\title{
Oncolytic HSV-1 rQNestin34.5v.2
}

National Cancer Institute

\section{Source}

National Cancer Institute. Oncolytic HSV-1 rQNestin34.5v.2. NCI Thesaurus. Code C139551.

A neuroattenuated, replication-competent, recombinant and genetically-eng ineered herpes simplex virus type 1 (HSV-1), with potential oncolytic and immunostimulating activities. In rQNestin34.5v.2, the UL39 gene encoding for the viral ribonucleotide reductase large subunit infected cell protein 6 (ICP6) and both endogenous copies of the gamma34.5 gene that encodes for the RL1 neurovirulence protein infected cell protein 34.5 (ICP34.5), which is needed for robust viral growth in an infected cell, are deleted, and one copy of the gamma34.5 gene is reinserted under control of a nestin promoter, which is selectively activated in gliomas. Upon intratumoral administration, oncolytic HSV-1 rQNestin34.5v.2 preferentially infects and replicates within the rapidly dividing, glioma cells, thereby directly lysing tumor cells. The released virus particles, in turn, infect and replicate in neighboring tumor cells, thereby further killing tumor cells. rQNestin34.5v.2 also elicits a tumor-specific systemic immune and cytotoxic T-lymphocyte (CTL) response, thereby killing nearby non-infected tumor cells. By inactivating UL39, viral ribonucleotide reductase activity is disrupted, resulting in the inhibition of nucleotide metabolism and viral DNA synthesis in non-dividing, healthy cells but not in dividing cells. Glioma-selective expression of ICP34.5 imparts tumor selectivity by preventing replication in healthy cells. 